\title{
Optimizing leading edge F-actin labeling using multiple actin probes, fixation methods and imaging modalities
}

$\overline{\text { Vera DesMarais }^{1,2,3, \star}, \text { Robert J Eddy1 }, \text { Ved P Sharma }}{ }^{1,3}$, Orrin Stone ${ }^{4}$ \& John S Condeelis ${ }^{1,2,3}$

\section{ABSTRACT}

We systematically evaluated the performance and reliability of several widely used, commercially available actinfilament probes in a highly motile breast adenocarcinoma cell line to optimize the visualization of F-actin-rich dynamic lamellipodia. We evaluated four Phalloidinfluorophores, two anti-actin antibodies, and three live-cell actin probes in five fixation conditions across three imaging platforms as a basis for the design of optimized protocols. Of the fluorescent phalloidin-dye conjugates tested, Alexa Fluor-488 Phalloidin ranked best in overall labeling of the actin cytoskeleton and maintenance of the fluorescence signal over time. Use of actin monoclonal antibodies revealed significant limitations under a variety of fixation-permeabilization conditions. Evaluation of commonly used live-cell probes provides evidence for actin filament bias, with TagRFPLifeact excluded from lamellipodia, but not mEGFP-Lifeact or F-tractin-EGFP.

\section{METHOD SUMMARY}

A strategy for optimizing F-actin labeling in dynamic lamellipodia of highly motile cells is given based on critical evaluation of five common fixation conditions, four Phalloidin-fluorophores, two anti-actin monoclonal antibodies, and three live-cell actin probes across three imaging platforms.

\section{KEYWORDS:}

actin - cell motility - F-tractin - lamellipodia $\cdot$ Lifeact $\cdot$ phalloidin

'Department of Anatomy \& Structural Biology, Albert Einstein College of Medicine, 1300 Morris Park Ave, Bronx, NY 10461, USA; ${ }^{2}$ Analytical Imaging Facility, Albert Einstein College of Medicine, Bronx, NY, USA; ${ }^{3}$ Gruss-Lipper Biophotonics Center, Albert Einstein College of Medicine, Bronx, NY, USA; ${ }^{4}$ Department of Pharmacology, UNC Chapel Hill School of Medicine, Chapel Hill, NC, USA; *Author for correspondence: vera.desmarais@einstein.yu.edu

BioTechniques 66: 113-119(March 2019) 10.2144/ btn-2018-0112
Actin is a highly abundant, conserved protein that exists in two principal forms: globular, monomeric (G) actin, and polymerized filamentous ( $F$ ) actin. Together with microtubules and intermediate filaments, actin filaments (F-actin) comprise the cytoskeleton of eukaryotic cells. Many cells harness actin polymerization to undergo directed migration or chemotaxis towards a soluble attractant source. This phenomenon of directed cell migration plays a central role during embryonic morphogenesis, wound healing, the inflammatory response and many disease states, including cancer metastasis [1,2]. During directed cell migration, newly polymerizing F-actin pushes the cell membrane outwards, forming such protrusive structures as lamellipodia and filopodia $[3,4]$. Subsequent steps include integrinmediated adhesion of the protrusion to the underlying extracellular matrix, generation of myosin II-dependent contractile forces on F-actin and selective de-adhesion and retraction of the cell [5].

F-actin in the cell periphery can be subdivided into the submembranous lamellipodium, consisting of a dynamic population of relatively short filaments and the adjacent lamella, containing longer, unbranched filaments stabilized by tropomyosin $[6,7]$. Due to the highly dynamic nature of F-actin, acquiring accurate and detailed images of the lamellipodium in both live and fixed cells can be challenging. The quality of images is directly related to the quality of the sample preparation, especially for quantitative high-resolution imaging modalities such as confocal and super-resolution microscopy.

Our study critically evaluates commonly used, commercially available fixation protocols and probes for labeling the actin cytoskeleton and guides the end user in making rational choices with respect to actin-probe selection. Specifically, our evaluation focused on: (1) the relative F-actin labeling quality and stability of commercially available fluorophores conjugated to phalloidin, a toxin derived from the Amanita phalloides mushroom that binds tightly to F-actin but not monomeric actin; (2) the impact of different fixation protocols on the accuracy and quality of F-actin labeling using commercial monoclonal antibodies; and (3) the accuracy and quality of $\mathrm{F}$-actin labeling using the live-cell actin probes Lifeact, F-tractin compared with phalloidin staining.

\section{MATERIALS \& METHODS \\ Cell culture}

A rat adenocarcinoma cell line, MTLn3, was cultured in MEM Alpha medium, cat. \#12561-056 (Life Technologies) supplemented with $5 \%$ fetal bovine serum, heat inactivated, cat. \# 100-106, (Gemini Bio-Products, CA, USA) and $0.5 \%$ penicillin/ streptomycin mixture (Life Technologies). 35-mm glass-bottom dishes, No. 1.5, cat. \# P35G-1.5-10-C, (MatTek Corporation, MA, USA) were incubated with MEM Alpha complete media for $30 \mathrm{~min}$ prior to plating. Approximately $1 \times 10^{5}$ MTLn3 cells at $30-50 \%$ confluency were then plated for $16 \mathrm{~h}$ at $37^{\circ} \mathrm{C}$ with $5 \% \mathrm{CO}_{2}$. For EGF-stimulation experiments, MTLn3 cells were starved for 3-4 h in Leibovitz's L15 media with no phenol red (Life Technologies) supplemented with $0.35 \%$ bovine serum albumin (BSA), Fraction $V$, cat. \# BP1600-100 (Fisher Chemical, PA, USA) at $37^{\circ} \mathrm{C}$ without $\mathrm{CO}_{2}$ followed by the addition of $5 \mathrm{nM}$ recombinant murine EGF, cat. \# PMG8044 (Life Technologies) in Leibovitz's L15 media supplemented with $0.35 \%$ BSA for $3 \mathrm{~min}$ at $37^{\circ} \mathrm{C}$.

\section{Fixation protocols}

Cells were rapidly rinsed $1 \mathrm{x}$ with Dulbecco's phosphate-buffered saline (dPBS) (Invitrogen, NY, USA) prior to fixation and permeabilization as follows: Sequential formaldehyde-triton protocol (sequential form-triton): $3.7 \%$ paraformaldehyde, EM 
$\checkmark$ grade, cat. \# 15700, (Electron Microscopy Sciences, PA, USA) in dPBS for $15 \mathrm{~min}$, rinsed $3 x$ with dPBS, followed by $0.15 \%$ TX-100 (Sigma-Aldrich, MO, USA) for 5 min; Simultaneous formaldehyde/glutaraldehyde/ saponin protocol (simultaneous form/glut/ saponin) [8] with modifications: Fix: 3.7\% paraformaldehyde, $0.1 \%$ glutaraldehyde, EM grade, cat. \#16000 (Electron Microscopy Sciences) and $0.15 \mathrm{mg} / \mathrm{ml}$ saponin, cat. \# 21435 (USB, OH, USA) in dPBS for $15 \mathrm{~min}$; Sequential formaldehyde-acetone protocol (sequential form-acetone): Fix with $3.7 \%$ paraformaldehyde in dPBS for $15 \mathrm{~min}$, rinsed $3 x$ with dPBS, followed by $-20^{\circ} \mathrm{C} 100 \%$ acetone, HPLC grade, cat. \# A949-1 (Fisher Chemical, PA, USA) for $5 \mathrm{~min}$ at $-20^{\circ} \mathrm{C}$; Methanol protocol: Fix with $-20^{\circ} \mathrm{C} 100 \%$ methanol for $10 \mathrm{~min}$; Acetone protocol: Fix with $-20^{\circ} \mathrm{C} 100 \%$ acetone for $10 \mathrm{~min}$.

For phalloidin staining, cells were rinsed $3 x$ with dPBS after Simultaneous Form/Glut/Saponin fixation, blocked for $30 \mathrm{~min}$ in $\mathrm{dPBS}+1 \% \mathrm{BSA}$ then incubated with 0.33 uM Alexa Fluor $^{\circledR} 488,555$ or 647 Phalloidin (Cell Signaling, Danvers, MA, USA) or $0.72 \mu \mathrm{M}$ CytoPainter Phalloidin-iFluor 405 Reagent (Abcam, MA, USA) in dPBS $+1 \%$ BSA for $15 \mathrm{~min}$. Cells were rinsed $3 x$ with dPBS and mounted in VectaShield Antifade, $\mathrm{H}-1000$ (Vector Laboratories, CA, USA) prior to imaging. For co-localization experiments using actin antibodies and phalloidin, cells were prepared for immunofluorescence by sequential form-triton, simultaneous form/ glut/saponin, sequential form-acetone, methanol and acetone protocol as described, blocked for $1 \mathrm{~h}$ in dPBS + 1\% BSA + 10\% normal goat serum (Cell Signaling, MA, USA) and incubated with a monoclonal antibody against a $\mathrm{N}$-terminal epitope of $\beta$-actin (clone AC-15), cat. \#ab6276 or a C-terminal epitope of actin (clone AC-40), cat. \#ab11003 (Abcam) in dPBS $+1 \%$ BSA for $1 \mathrm{~h}$ at $1: 400$ or 1:200 dilution, respectively. Cells were rinsed $3 x$ with $\mathrm{dPBS}+1 \% \mathrm{BSA}$ and incubated with Goat anti-Mouse IgG $(\mathrm{H}+\mathrm{L})$ cross-adsorbed secondary antibody conjugated to Alexa Fluor 555, cat. \#A-21422 (Life Technologies) at 1:300 dilution for $1 \mathrm{~h}$. Cells were rinsed three times with dPBS, incubated with 0.33 $\mu \mathrm{M}$ Alexa Fluor 488 phalloidin for $15 \mathrm{~min}$ in $\mathrm{dPBS}+1 \% \mathrm{BSA}$, rinsed $3 x$ with dPBS and mounted as described. For co-localization using transient transfected FP-Lifeact and phalloidin, cells were grown to $30-50 \%$ confluency, rinsed with Opti-MEM and transfected for $1.5 \mathrm{hr}$ with Lipofectamine 2000 (Life Technologies) + Opti-MEM (Life Technologies) using manufacturer's instructions. Cells were allowed to recover in MEM Alpha complete media for $2 \mathrm{~h}$, treated with 0.05\% Trypsin-EDTA, cat. \#25300-054 (Life Technologies) for $5 \mathrm{~min}$ and rinsed before plating. Cells were allowed to express mEGFP-Lifeact, cat. \#54610, TagRFPLifeact, cat. \#54586 or F-tractin-EGFP, cat. \#58473 (Addgene, MA, USA) for $16 \mathrm{~h}$ at $37^{\circ} \mathrm{C}$ with $5 \% \mathrm{CO}_{2}$ before simultaneous form/glut/ saponin fixation and staining with $0.33 \mu \mathrm{M}$ of Alexa Fluor 555-phalloidin or $0.33 \mu \mathrm{M}$ of Alexa Fluor 488-phalloidin, respectively.

\section{Image acquisition \& processing}

Wide-field images were acquired on a GE DeltaVision microscope with a $60 x$ objective (N.A. 1.42) and a CoolsnapHQ2 camera (Photometrics). Confocal images were acquired on a Leica SP5 and Leica SP8 pointscanning confocal microscope using the $63 x$ objective (N.A. 1.4), zoom = 2, line averaging $=4$. All images represent maximal pixel-projections of z-series, step size $=0.2 \mu \mathrm{m}$. Structured illumination microscopy (SIM) images were acquired on a Nikon 3D-SIM microscope with a 100x objective (N.A. 1.49) and an EMCCD Andor iXon3 DU897 camera, using Nikon Elements software for SIM processing. All images represent maximal pixel-projections of z-series, step size $=0.12 \mu \mathrm{m}$.

\section{Image analysis}

To quantify the change in fluorescence of different phalloidin conjugates during shortterm storage, phalloidin stained cells were imaged at various time points for 13 days using widefield microscopy. In order to avoid any photobleaching effects, three previously not imaged fields-of-view were imaged at each time point. Fluorescence intensities were measured in ImageJ after finding the boundaries of cells by setting a threshold and normalized by dividing each value by the day-1 fluorescence intensity, and multiplying by 100 to show percent fluorescent intensities over time. Fluorescence intensity linescans were performed in ImageJ and measured along the line (length $=18.4 \mu \mathrm{m}$, width $=0.8 \mu \mathrm{m}$ ) starting from the outside of the cell to the cell interior, capturing the leading edge and stress fibers. Fluorescence intensity values were normalized by the maximum value.

\section{RESULTS \& DISCUSSION}

Staining with fluorescently conjugated Phalloidin is considered the most reliable method of accurately labeling F-actin in fixed eukaryotic cells $[9,10]$. However, our preliminary observations indicated that different fluorescent dye conjugates of phalloidin labeled the actin cytoskeleton with varying degrees of resolution and quality. In order to document these differences, rat breast adenocarcinoma MTLn3 cells put through a simultaneous form/glut/saponin fixation and permeabilization protocol were labeled with phalloidin conjugated with iFluor 405 (Phalloidin-405), Alexa Fluor 488 (Phalloidin-488), Alexa Fluor 555 (Phalloidin-555) and Alexa Fluor 647 (Phalloidin-647). This protocol has proven successful in localizing F-actin by phalloidin staining in MTLn3 cells [11] and motile human neutrophils [8]. Initial wide-field imaging revealed positive labeling of actin structures including lamellipodia, filopodia and stress fibers for all four dye-conjugates of phalloidin (Figure 1A, row 1). However, images acquired by confocal (Figure 1A, rows 2 and 3 ) and super-resolution microscopy (SIM) (Figure $1 \mathrm{~A}$, rows 4 and 5 ), uncovered profound differences. Cells labeled with Phalloidin-488 showed highly detailed actin-filament labeling and displayed the best overall image quality, followed closely by Phalloidin- 555 . However, images acquired with Phalloidin- 405 and phalloidin- 647 were less detailed and of inferior quality compared with phalloidin-488. Phalloidin-405 appeared to give inferior staining quality across all imaging platforms, possibly due to inherent binding properties of the dye. Phalloidin-647 works well for epifluorescence and confocal, but not SIM, perhaps because Alexa647 is easily pushed to a Triplet Dark State by laser illumination, making acquisition of the many frames of evenly illuminated images needed for SIM difficult, resulting in inferior SIM images [12].

Preliminary imaging of F-actin uncovered wide variation in the ability of phalloidinfluorophores to maintain their signal during short-term storage. To quantify these observations, MTLn3 cells underwent simultaneous form/glut/saponin fixation, stained with phalloidin conjugated to various 
fluorophores and immediately imaged by wide-field fluorescent microscopy to obtain a baseline fluorescent signal (day 1). Samples were stored at $4^{\circ} \mathrm{C}$ in the dark and different fields of cells were imaged at day 5,8 and 13 under identical exposure conditions. Phalloidin-488 and Phalloidin-647 maintained their fluorescence at day 5 , 8 and 13. However, Phalloidin-555 rapidly lost nearly $70 \%$ of its fluorescence by day 5. Similarly, Phalloidin- 405 lost $30 \%$ of its fluorescence signal by day 5 and gradually faded to nearly $0 \%$ by day 13 (Figure 1B). These observations demonstrate the choice of fluorophore attached to Phalloidin is a critical factor for fixed cells that require short-term storage, with Phalloidin-488 being superior in image quality and resistance to fluorescence signal loss over time.

The development of a fixation protocol for studying dynamic lamellipodia in highly motile cells requires a trade-off between speed, morphology and antigen preservation, so in order to maximize the quality of staining, we examined several fixation methods, including aldehydes and organic solvents. Although aldehydes preserve cellular morphology far better than organic solvents, they act rather slowly compared to organic solvents. To determine which fixation method best captures the F-actin within dynamic lamellipodia, MTLn3 cells were stimulated with EGF and labeled with Phalloidin-488 after fixation (Figure 2A, top row). We found the simultaneous form/glut/saponin protocol greatly enhanced the staining of F-actin in lamellipodia, compared with the slower sequential form-triton protocol (Figure 2A, top row, \& Figure 2B, top row). Simultaneous form/glut/saponin also had superior preservation of actin morphology under SIM (Figure 3), with strong labeling of the leading edge and interior actin filaments. Sequential formaldehyde fixation followed by acetone permeabilization (sequential form-acetone) also showed enhanced lamellipodial F-actin staining but resulted in inferior cell morphology with cells appearing retracted and ruffled (compare Figure 2A, top row). Methanol or acetone fixation showed inferior staining with Phalloidin-488, giving a dotted, disrupted staining pattern both at lamellipodia and in stress fibers, confirming previous studies that show methanol fixation as unsuitable

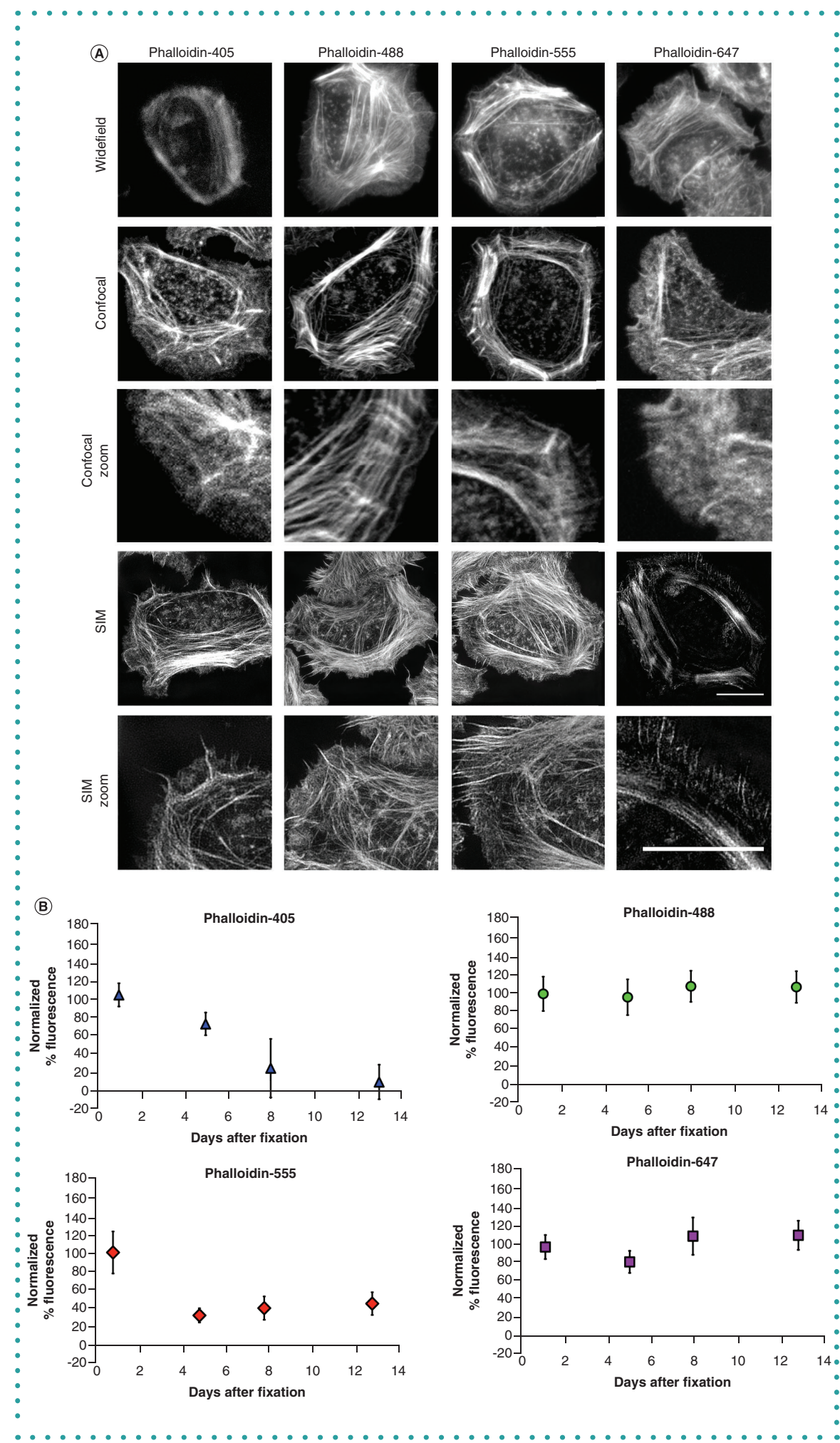

Figure 1. Staining cells with Phalloidin- 488 reveals the most detailed actin structures and is stable over time. MTLn3 cells were fixed by simultaneous form/glut/saponin and stained with Phalloidin-405, Phalloidin-488, Phalloidin-555 and Phalloidin-647. (A) Images were acquired by widefield fluorescence, confocal and SIM super-resolution microscopy. Images were acquired as z-stacks and displayed as maximal pixel-projections. Scale bar: $10 \mu \mathrm{m}$. (B) The persistence of fluorescent signal in fixed samples over 2 weeks. Fluorescent intensities of cells of the same fixed sample were quantified by acquiring images via widefield fluorescence on four different days within a 2-week period. Fluorescent intensities are plotted as percent fluorescent intensity normalized to day 1. SIM: Structured illumination microscopy. 


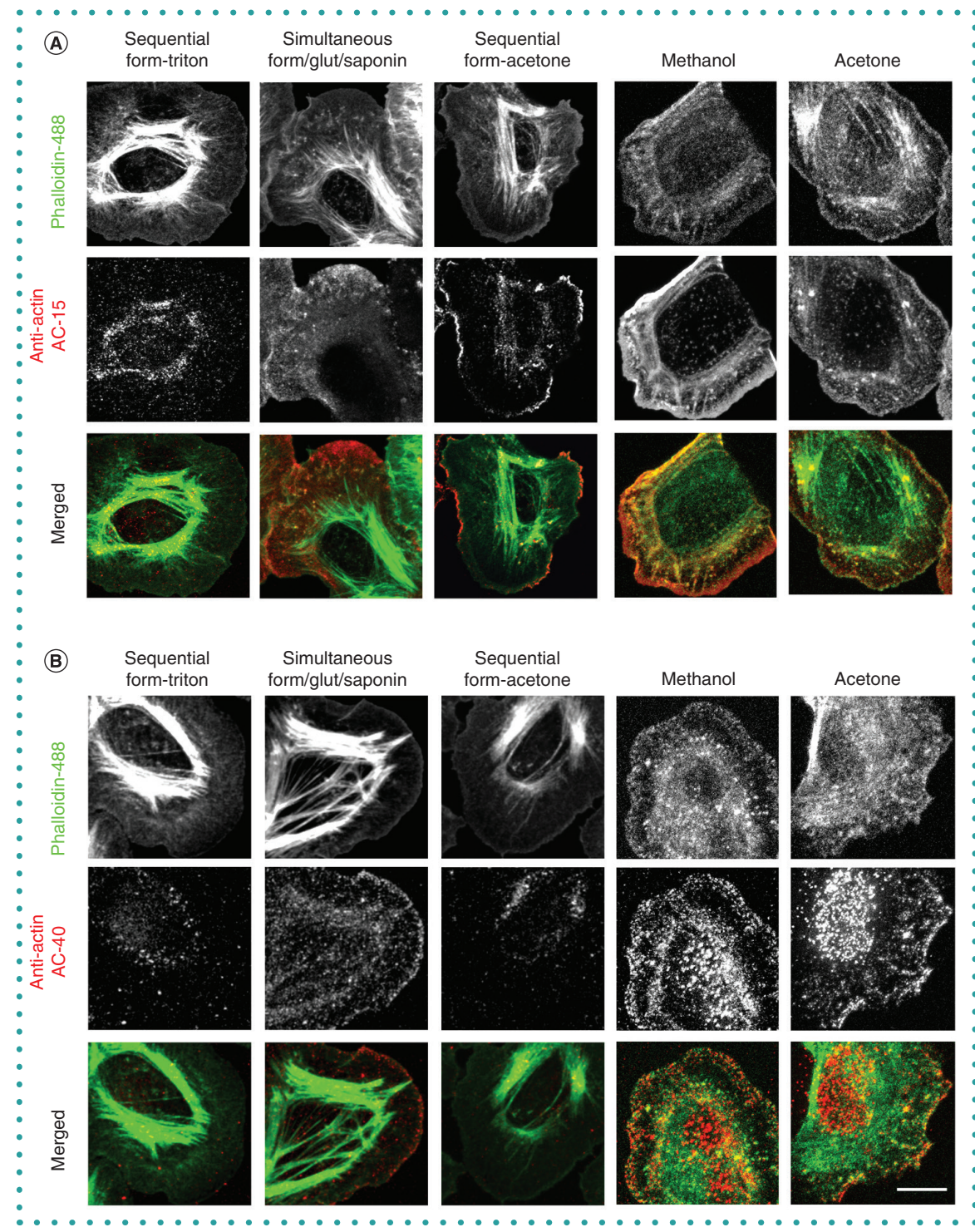

Figure 2. Labeling of different cellular actin compartments with actin antibodies is highly dependent on fixation method. MTLn3 cells were treated with EGF for 3 min, fixed using five different fixation methods as indicated, and incubated with either (A) anti-actin AC-15 or (B) antiactin AC-40 followed by incubation with Alexa 555 goat anti-mouse secondary antibody, then stained with Phalloidin-488. All images were acquired via confocal microscopy and are displayed as maximal pixel-projections of z-stacks. Scale bar: $10 \mu \mathrm{m}$.

$\checkmark$ for phalloidin-staining due to destruction of the native conformation of F-actin [13].

The superiority of the simultaneous form/glut/saponin protocol in preserving labile F-actin structures such as lamellipodia over the other fixation protocols tested (Figure 3 ) most likely results from the fact that Triton X-100 is a nonselective detergent, extracting both proteins and lipids from the plasma membrane while saponin is a gentler detergent that selectively interacts with plasma membrane cholesterols to form pores. This property of saponin may contribute to greater preser- clone AC-15, directed against a N-terminal $\beta$-actin epitope, labeled the EGF-induced lamellipodium of MTLn3 cells under all fixation conditions except sequential form-triton (Figure 2A). By contrast, clone AC-40, directed against a C-terminal pan-actin epitope, failed to label lamellipodia with sequential form-triton as well as sequential form-acetone fixation. Positive staining of lamellipodia by clone AC-40 was only observed with simultaneous form/glut/saponin, methanol and acetone fixation (Figure 2B). Despite the presence of a positive signal at lamellipodia after methanol fixation using AC-15 and AC-40 by confocal microscopy, closer inspection by SIM revealed images of inferior quality and poor F-actin morphology, as shown in Figure 4

Surprisingly, AC-15 and AC-40 labeled stress fibers poorly under all fixation conditions, with or without the addition of phalloidin (data not shown), including methanol and acetone fixation recommended by commercial vendors. While the poor staining of stress fibers may be cell-type specific, our analysis suggests caution when using actin monoclonal antibodies to label various actin compartments in cells.

In order to label F-actin in nonpermeabilized live cells, a variety of genetically encoded F-actin probes fused to fluorescent protein tags have been developed, for example Lifeact, an actin-binding peptide derived from yeast ABP140 [14] and F-tractin, an actin-binding peptide derived from inositol 1,4,5-triphosphate 3-kinase A or ITPKA $[15,16]$. We tested how these genetically encoded F-actin probes compare with phalloidin labeling in fixed lamellipodia. Fluorescence intensity line-scans demonstrate that mEGFP-Lifeact and F-tractin-EGFP highly co-localize with Phalloidin- 555 at both leading edge lamellipodia and stress fibers. By contrast, TagRFP-Lifeact co-localized with Phalloidin-488 at stress fibers but was nearly absent from lamellipodia (Figure 5). These results support the previous observations of $\mathrm{F}$-actin bias when using live-cell probes $[15,17]$ and suggest the cellular distribution of individual Lifeact fluorescent protein reporters do not always accurately represent the actin cytoskeleton in a given cell type. 


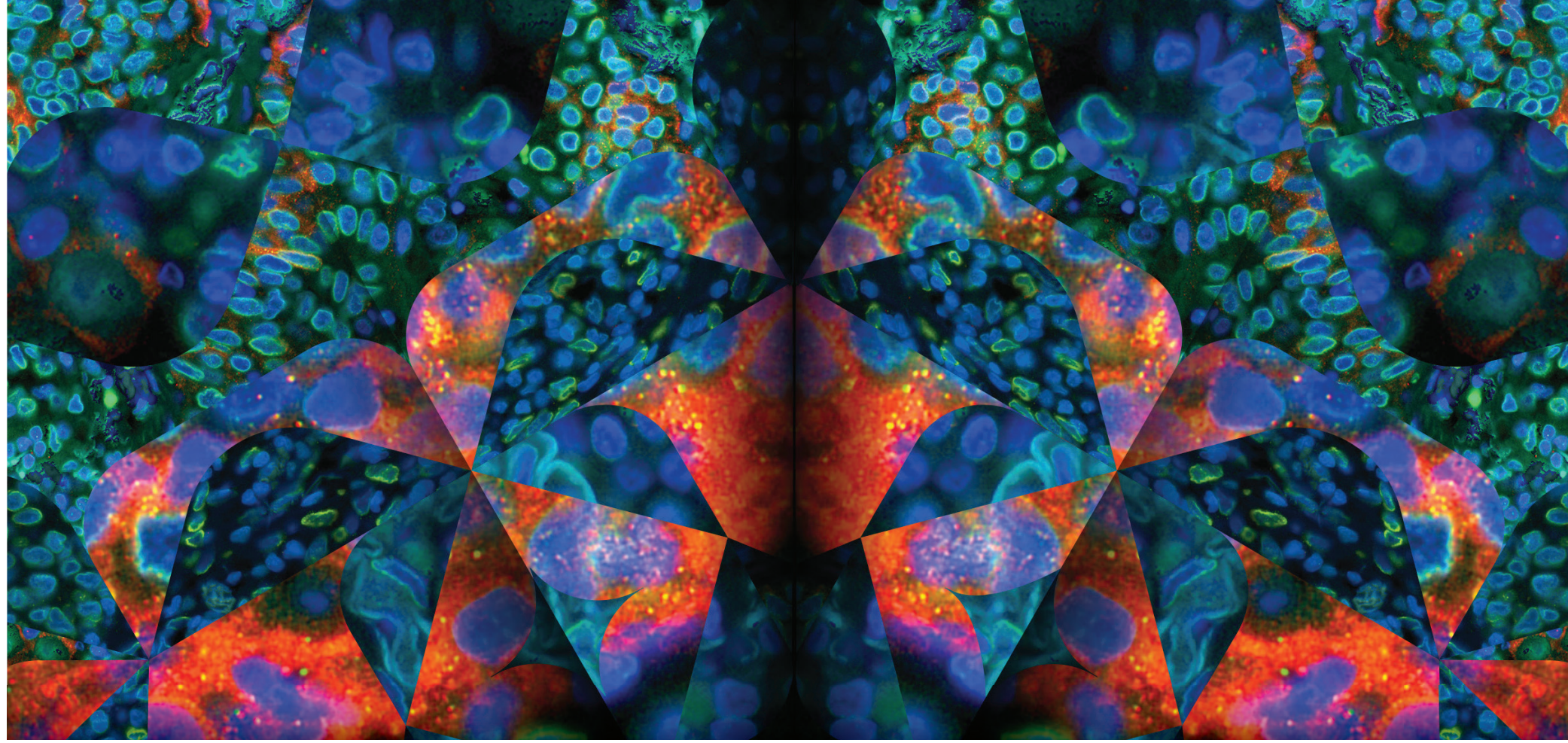

\section{Brilliant visualization}

\section{See the difference to make big breakthroughs in your tumor microenvironment research}

In the world of next generation immuno-oncology research, having confidence in your immunoassay results is vital. Unfortunately, $75 \%$ of antibodies in today's market are non-specific or simply do not work at all.* That's why at Bethyl, we manufacture and validate every antibody on-site to ensure target specificity and sensitivity. More than 10,000 independent citations over the past 15 years have proven that our antibodies will function as designed in your assay - and we offer a 100\% guarantee. Work with Bethyl to bring your discovery into full focus.

\section{See our data at bethyl.com/immuno-oncology}
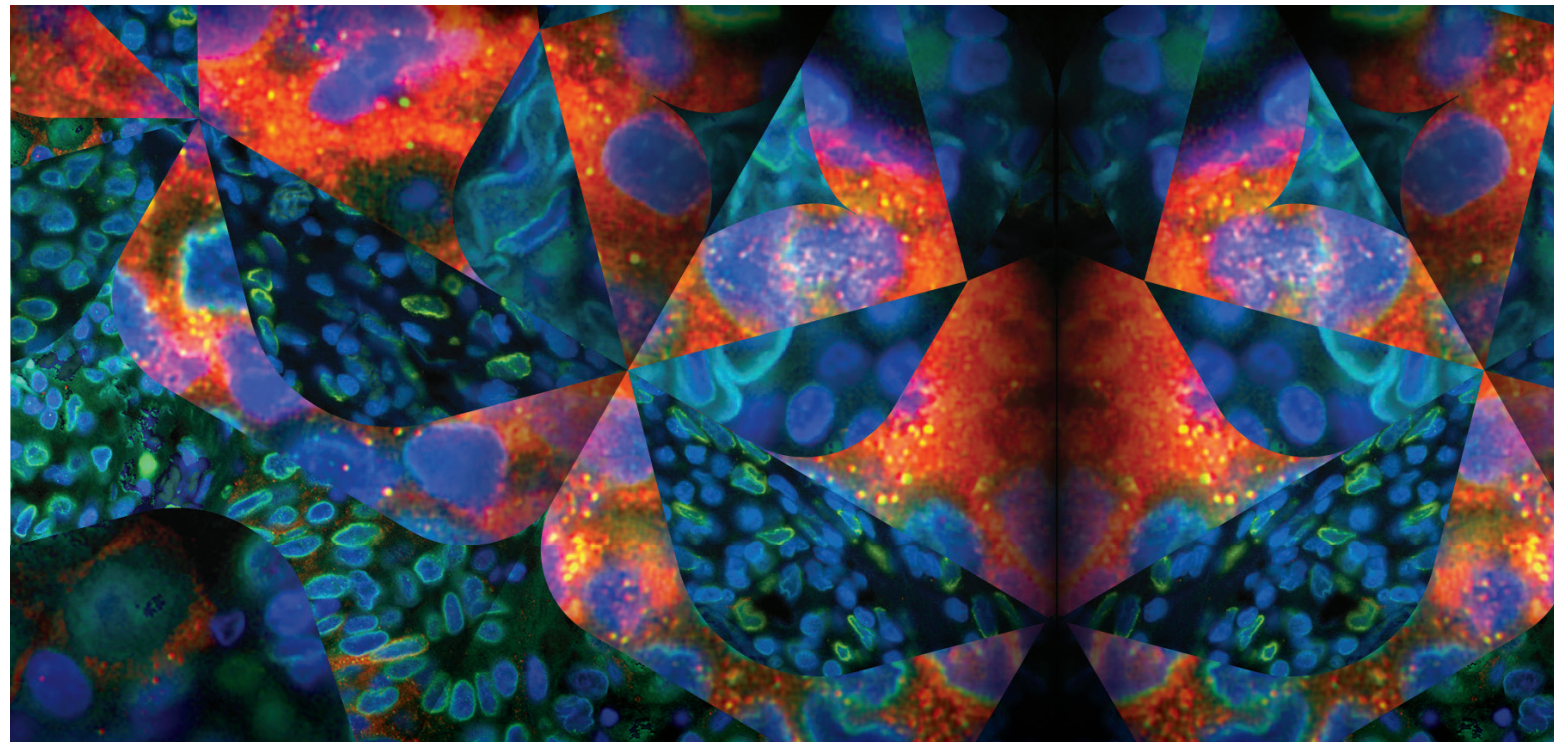

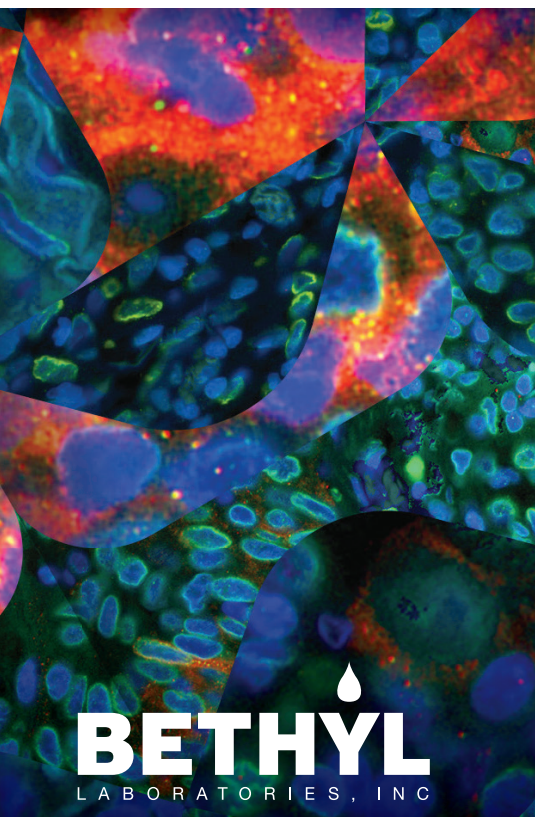

Really Good Antibodies 


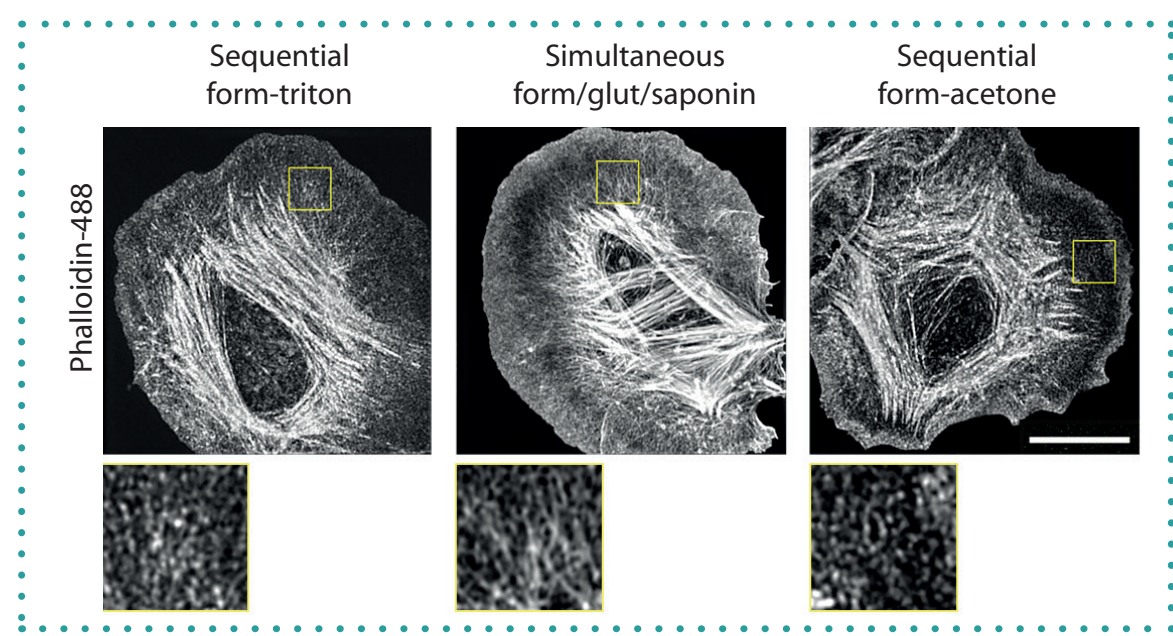

Figure 3. Super-resolution imaging (structured illumination microscopy) of Phalloidin-488-labeled cells shows highly detailed actin filaments under simultaneous form/glut/saponin fixation. MTLn3 cells were treated with EGF for $3 \mathrm{~min}$, fixed as indicated and stained with Phalloidin-488. Images were acquired by structured illumination microscopy and displayed as maximal pixel-projections of z-stacks. Zoomed insets (yellow box) show actin filament staining from similar regions of the lamellipodium. Scale bar: $10 \mu \mathrm{m}$.

- After testing three different labeling modalities for F-actin with a variety of different fixation techniques, phalloidin was judged to be the most robust method of labeling. Specifically, we found Phalloidin-488 to give the most detailed, stable staining, under imaging conditions ranging from widefield to confocal to SIM. The actin cytoskeleton appeared to be preserved best by rapid, simultaneous form/ glut/saponin fixation. Antibody staining with AC-15 and AC-40 did not reveal the same level of detail of the actin structures as phalloidin staining and showed vast differ- ences of staining patterns under different fixation conditions. Based on their colocalization with phalloidin, positive labeling of actin at the lamellipodium can be achieved in live cells expressing mEGFP-Lifeact and F-tractin-EGFP. However, TagRFP-Lifeact did not show localization at lamellipodia, suggesting not all live-cell actin reagents are equally useful for F-actin labeling.

\section{FUTURE PERSPECTIVE}

Phalloidin staining as well as actin antibody labelling and expression of genetically encoded probes such as Lifeact have been routinely used to visualize the actin cytoskeleton in fluorescence microscopy. While these techniques have been sufficient when labeling broad actin structures such as stress fibers, labeling dynamic actin compartments has been particularly challenging. As microscope technology has evolved from epifluorescence to confocal to SIM superresolution, pushing to visualize smaller cellular structures at higher resolution; and as researchers investigate finer and more detailed actin networks in cellular protrusions, it has become apparent that routine staining techniques do not necessarily label these structures under standard conditions. As we have shown in this paper, the acquisition of high-quality images of dynamic F-actin compartments depends on sample preparation and requires a critical evaluation of standard methods for each cell type. In the future, it will be critically important that newly developed actin probes are stable over time, show dense, uniform labeling along all actin structures and have the ability to accurately report actin filaments in all compartments while avoiding perturbation of a cell's actin regulatory mechanisms.

\section{AUTHOR CONTRIBUTIONS}

VD, RE and VS conceived the study and contributed equally. RE conducted the cell staining experiments. VD acquired all images and conducted data analysis. VS provided preliminary observations that the study is based on and conducted data analysis. VD, RE, VS and JC co-wrote the
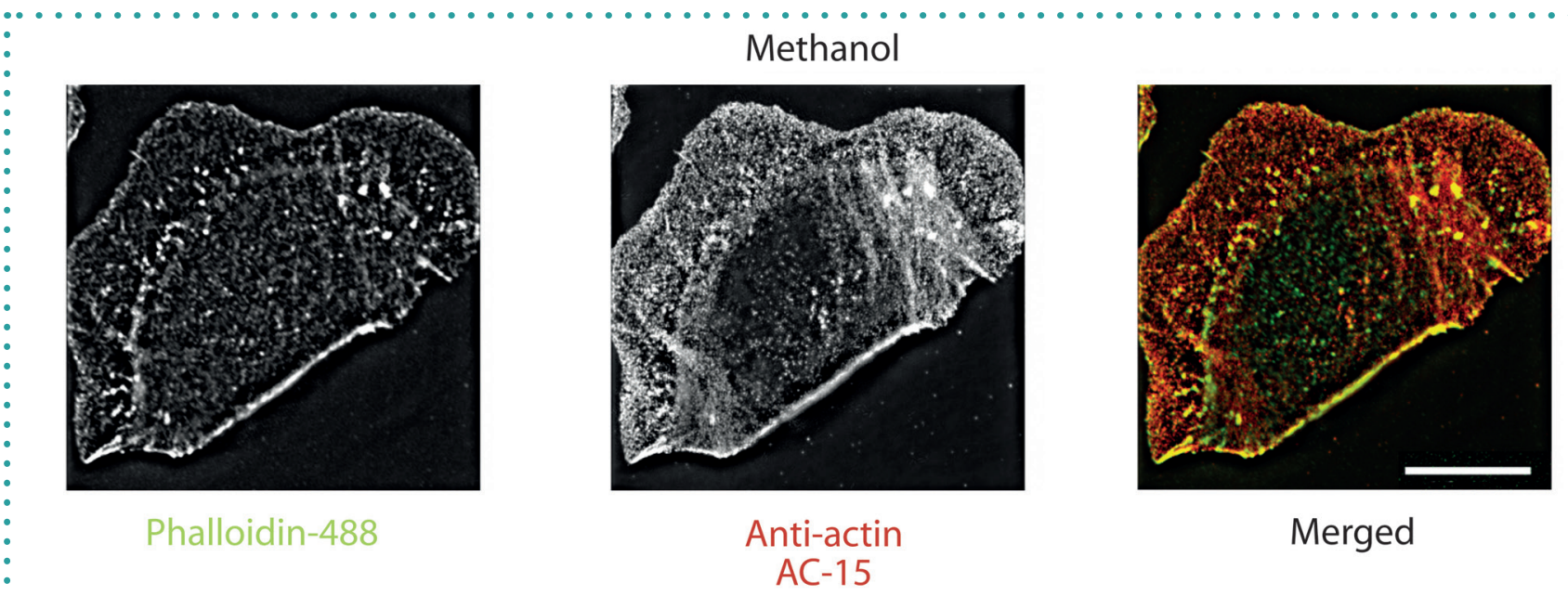

Merged

Figure 4. Structured illumination microscopy imaging of cells stained with anti-actin AC-15 after methanol fixation. MTLn3 cells were treated with EGF for $3 \mathrm{~min}$, fixed with methanol, incubated with anti-actin AC-15 followed by incubation with Alexa 555 goat anti-mouse secondary antibody, then stained with Phalloidin-488. Images were acquired by structured illumination microscopy and displayed as maximal pixel-projections of z-stacks. Scale bar: $10 \mu \mathrm{m}$. 
manuscript. OS provided preliminary results and materials.

\section{FINANCIAL \& COMPETING INTERESTS DISCLOSURE}

All imaging was conducted in the Analytical Imaging Facility (AIF) (funded by $\mathrm{NCI}$ Cancer Grant P30CA013330). SIM imaging was executed on the Nikon-STORM/SIM/ TIRF microscope (funded by $\mathrm{NIH}$ 1S100D18218-1). Some confocal imaging was executed on the Leica SP8 confocal microscope (funded by $\mathrm{NIH}$ 1S100D023591-01). The research was funded by NIH grants CA150344 and CA216248 (RE, VS, JC). The authors have no other relevant affiliations or financial involvement with any organization or entity with a financial interest in or financial conflict with the subject matter or materials discussed in the manuscript apart from those disclosed.

No writing assistance was utilized in the production of this manuscript.

\section{OPEN ACCESS}

This work is licensed under the AttributionNonCommercial-NoDerivatives 4.0 Unported License. To view a copy of this license, visit http://creativecommons.org/licenses/ by-nc-nd/4.0/

\section{REFERENCES}

1. Franz CM, Jones GE, Ridley AJ. Cell migration in development and disease. Dev. Cell 2(2), 153-158 (2002).

2. Roussos ET, Condeelis JS, Patsialou A. Chemotaxis in cancer. Nature Rev. Cancer 11(8), 573-587 (2011).

3. Condeelis $\mathrm{J}$. Life at the leading edge: the formation of cell protrusions. Annu. Rev. Cell Biol. 9, 411-444 (1993).

4. Wear MA, Schafer DA, Cooper JA. Actin dynamics: assembly and disassembly of actin networks. Curr. Biol. 10(24), R891-R895 (2000).

5. Lauffenburger DA, Horwitz AF. Cell migration: a physically integrated molecular process. Cell $84(3), 359-369$ (1996).

6. Svitkina TM, Borisy GG. Arp2/3 complex and actin depolymerizing factor/cofilin in dendritic organization and treadmilling of actin filament array in lamellipodia. J. Cell Biol. 145(5), 1009-1026 (1999).

7. Desmarais V, Ichetovkin I, Condeelis J, Hitchcock-Degregori SE. Spatial regulation of actin dynamics: a tropomyosin-free, actin-rich compartment at the leading edge. J. Cell Sci. 115(Pt 23), 4649-4660 (2002).

8. Pierini LM, Lawson MA, Eddy RJ, Hendey B, Maxfield FR. Oriented endocytic recycling of alpha5beta1 in motile neutrophils. Blood 95(8), 2471-2480 (2000).

9. Vandekerckhove J, Deboben A, Nassal M, Wieland T. The phalloidin binding site of F-actin. EMBO J. 4(11) 2815-2818 (1985)

10. Melak M, Plessner M, Grosse R. Actin visualization at glance. J. Cell Sci. 130(3), 525-530 (2017).

11. Song $X$, Chen $X$, Yamaguchi $H$, Mouneimne G, Condeelis JS, Eddy RJ. Initiation of cofilin activity in response to EGF is uncoupled from cofilin phosphorylation and dephosphorylation in carcinoma cells. J. Cell Sci. 119(Pt 14), 2871-2881 (2006).

12. Dempsey GT, Vaughan JC, Chen KH, Bates M, Zhuang X. Evaluation of fluorophores for optimal performance in localization-based super-resolution imaging. Nat. Methods 8(12), 1027-1036 (2011).

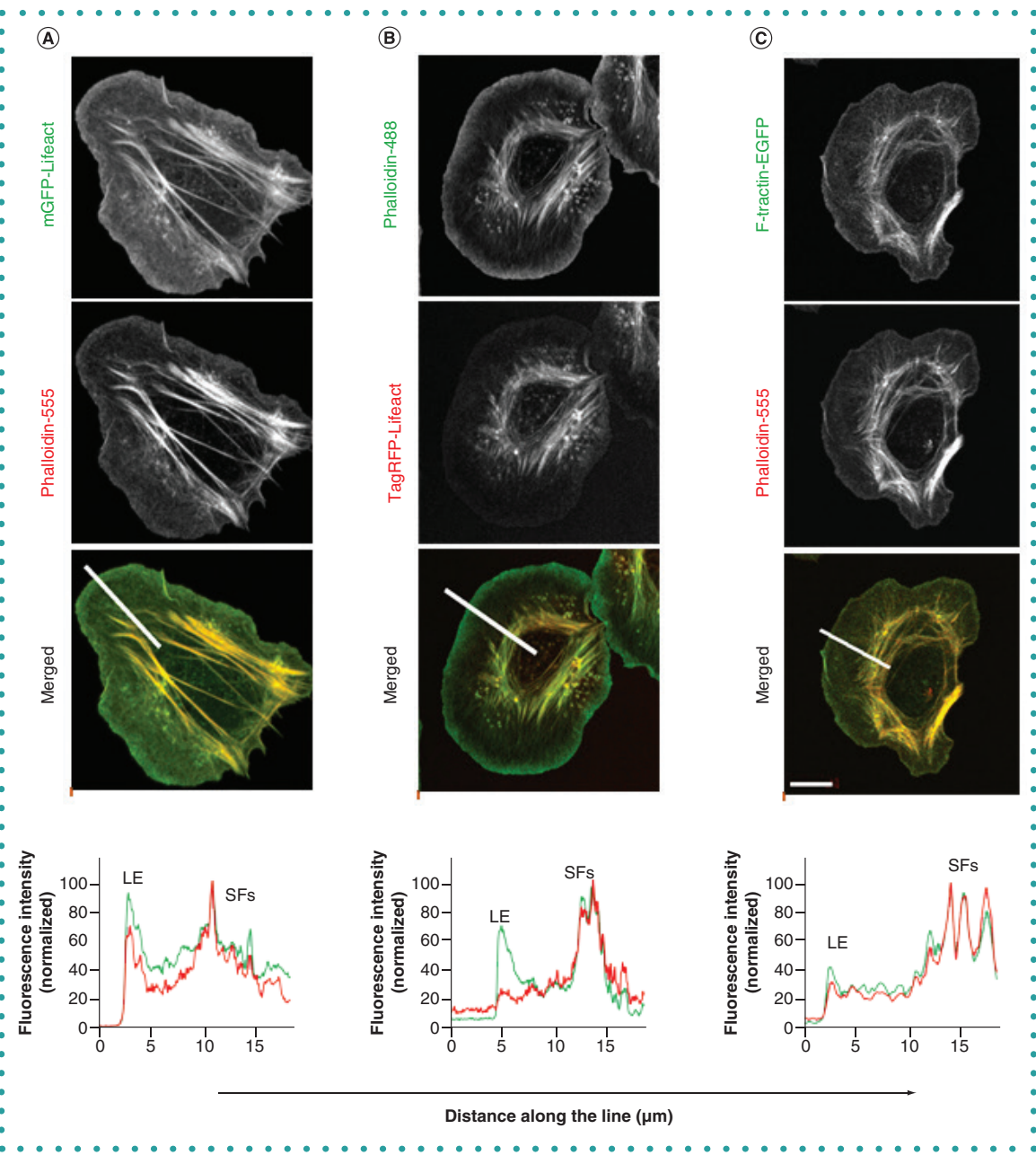

Figure 5. mEGFP-Lifeact and F-tractin-EGFP show actin-labeling patterns similar to phalloidin staining, but TagRFP-Lifeact does not. MTLn3 cells were transfected with Lifeact and F-tractin plasmids, treated with EGF for 3 min, fixed with simultaneous form/glut/saponin, stained with phalloidin and then imaged. (A) mEGFP-Lifeact and Phalloidin-555 (B) TagRFP-Lifeact and Phalloidin-488 (C) F-tractin-EGFP and Phalloidin-555. >100 cells, $n=3$ were visually examined by confocal microscopy for each condition $(A, B, C)$ and a representative cell was chosen for analysis. Graphs are fluorescent intensity quantifications along the line indicated in the merged image. All confocal images are displayed as maximal pixel-projections of z-stacks. Scale bar: $10 \mu \mathrm{m}$ LE: Leading edge; SF: Stress fiber.

13. Kellogg DR, Mitchison TJ, Alberts BM. Behaviour of microtubules and actin filaments in living Drosophila embryos. Development 103(4), 675-686 (1988)

14. Riedl J, Crevenna AH, Kessenbrock K et al. Lifeact: a versatile marker to visualize F-actin. Nat. Methods 5(7), 605-607 (2008)

15. Belin BJ, Goins LM, Mullins RD. Comparative analysis of tools for live cell imaging of actin network architecture. Bioarchitecture 4(6), 189-202 (2014).

16. Schell MJ, Erneux C, Irvine RF. Inositol 1,4,5-trisphosphate 3-kinase $A$ associates with F-actin and dendritic spines via its $\mathrm{N}$ terminus. J. Biol. Chem. 276(40), 37537-37546 (2001)

17. Sanders TA, Llagostera E, Barna M. Specialized filopodia direct long-range transport of SHH during vertebrate tissue patterning. Nature 497(7451), 628-632 (2013). 